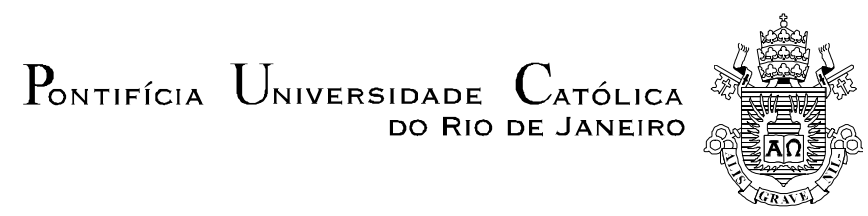

André Fichel Nascimento

\title{
Avaliação de Investimentos em Tecnologia da Informação: uma Perspectiva de Opções Reais
}

\author{
Dissertação de Mestrado
}

Dissertação apresentada ao Programa de Pós-graduação em Engenharia Industrial da PUC-Rio como requisito parcial para obtenção do título de Mestre em Engenharia Industrial.

Orientador: Tara Keshar Nanda Baidya

Rio de Janeiro

Abril de 2005 


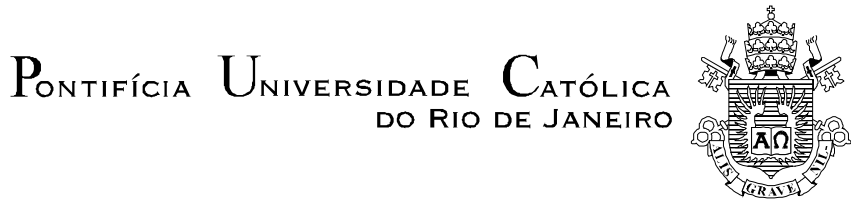

\section{André Fichel Nascimento}

\section{Avaliação de Investimentos em Tecnologia da Informação: uma Perspectiva de Opções Reais}

Dissertação apresentada como requisito parcial para a obtenção do grau de Mestre pelo Programa de Pós-graduação em Engenharia Industrial da PUC-Rio. Aprovada pela Comissão Examinadora abaixo assinada.

Prof. Tara Keshar Nanda Baidya

Orientador

DEI - PUC-Rio

Prof. José Paulo Teixeira

DEI - PUC-Rio

Prof. Carlos Patrício Samanez

DEI - PUC-Rio

Prof. José Eugênio Leal

Coordenador Setorial do Centro

Técnico Científico - PUC-Rio

Rio de janeiro, 11 de Abril de 2005 
Todos os direitos reservados. É proibida a reprodução total ou parcial do trabalho sem autorização da universidade, do autor e do orientador.

\section{André Fichel Nascimento}

Graduou-se em Engenharia da Computação na PUC-Rio em Agosto de 2001. Durante o período de graduação, estagiou em diversas empresas e foi bolsista CNPQ da empresa Espaço $\mathrm{A}+\mathrm{C}$ e bolsista IBM do TecComm, grupo de pesquisas em comércio eletrônico, na PUC-Rio. Após a graduação foi consultor da Advus/Lumina durante quase dois anos e capacitou-se em Gerência de Projetos pela Fundação Vanzolini na USP em 2002. Possui interesse nas áreas de Informática, Finanças e Gerência de Projetos.

Ficha Catalográfica

Nascimento, André Fichel

Avaliação de investimentos em tecnologia da informação : uma perspectiva de opções reais / André Fichel Nascimento ; orientador: Tara Keshar Nanda Baidya. - Rio de Janeiro : PUC, Departamento de Engenharia Industrial, 2005.

151 f. : il. ; $30 \mathrm{~cm}$

Dissertação (mestrado) - Pontifícia Universidade Católica do Rio de Janeiro, Departamento de Engenharia Industrial.

Inclui referências bibliográficas.

1. Engenharia Industrial - Teses. 2. Avaliação de projetos. 3. Opções reais. 4. Simulação de Monte Carlo. 5. Regressão dos mínimos quadrados. 6. Tecnologia da informação. I. Baidya, Tara Keshar Nanda. II. Pontifícia Universidade do Rio de Janeiro. Departamento de Engenharia Industrial. III. Título. 


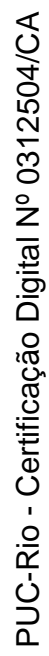

Aos meus pais, que sempre me ajudaram, dando-me muita força e incentivo 


\section{Agradecimentos}

A Deus, por me dar esta oportunidade.

Aos meus pais, pelo amor, carinho, educação, paciência e todo apoio dado durante o mestrado.

Ao meu orientador, Professor Tara Keshar Nanda Baidya, pelo estimulo, pela paciência, pela amizade e pela colaboração neste trabalho.

Aos meus professores José Paulo Teixeira, Carlos Patrício Samanez e Leonardo Lima pelos ensinamentos dados.

A Marcelo Sant'Anna, pelo estímulo e direcìonamento.

Aos meus amigos e colegas de estudo, pela companhia e pela amizade.

A todos os meus familiares que de alguma forma me ajudaram ou me estimularam.

A todos os funcionários do Departamento de Engenharia Industrial, por tornar a minha vida mais fácil.

À CAPES e ao Departamento de Engenharia Industrial da PUC-Rio, pelos auxílios concedidos, sem os quais este trabalho não poderia ter sido realizado. 


\section{Resumo}

Nascimento, André Fichel; Baidya, Tara Keshar Nanda. Avaliação de Investimentos em Tecnologia da Informação: uma Perspectiva de Opções Reais. Rio de Janeiro, 2005. 151p. Dissertação de Mestrado Departamento de Engenharia Industrial, Pontifícia Universidade Católica do Rio de Janeiro.

Com o crescimento da participação da indústria tecnológica na economia global durante a última década, a avaliação de investimentos em tecnologia de informação, ou TI, vem merecendo cada vez mais atenção por profissionais e estudiosos de finanças. No entanto, devido às características dinâmicas e incertas dos projetos de TI, as ferramentas tradicionais de orçamentação de capital não são adequadas para avaliar este tipo de investimento. Por outro lado, a aplicação do método das opções reais permite uma forma de se quantificar a geração de valor em investimentos com características semelhantes aos investimentos em TI. Este estudo visa justamente aplicar a teoria das opções reais na avaliação de investimentos em tecnologia da informação. Para isso, relacionam-se dois dos principais modelos de avaliação de investimentos em tecnologia da informação, baseados em opções reais. Os modelos adotados levam em conta as incertezas nos custos e nos benefícios do projeto, além da possibilidade da ocorrência de um evento catastrófico, que proporcionaria o fim do projeto. No presente trabalho, avalia-se a decisão de investimento tanto para projetos de pesquisa e desenvolvimento quanto para projetos de aquisição e desenvolvimento de ativos de TI. Uma atenção especial foi dada à solução numérica dos modelos. Métodos numéricos baseados em simulação de Monte Carlo foram analisados e utilizados para resolver os exemplos.

\section{Palavras-chave}

Avaliação de Projetos; Opções Reais; Simulação de Monte Carlo; Regressão dos Mínimos Quadrados; Tecnologia da Informação (TI). 


\section{Abstract}

Nascimento, André Fichel; Baidya, Tara Keshar Nanda. Valuation of Information Technology Investments: a Real Options Perspective. Rio de Janeiro, 2005. 151p. MSc. Dissertation - Departamento de Engenharia Industrial, Pontifícia Universidade Católica do Rio de Janeiro.

Based on the growth of the technological industry's share of the economy during the last decade, the valuation of information technology (IT) investments has recently been receiving more and more attention. However, due to the dynamic and uncertain features of IT projects, traditional capital budgeting techniques do not apply to this type of investment. On the other hand, putting in practice real option valuation enables quantification of the value generated by investments with similar characteristics of IT investments. This study does just that, by applying the real options theory to value information technology investments. In order to achieve this, a couple of models based on real options and applied to IT investment valuation were considered. The approach takes into account uncertainty in costs and benefits to be generated, as well as the possible occurrence of catastrophic events, which could put an end to the project. In the current work, the model presented can be used to value resource and development projects as well as IT acquisition and development projects. Special attention has been given to the numerical solution of the models, where numerical methods based on Monte Carlo simulation were first analyzed and then used to solve the examples.

\section{Keywords}

Project Valuation; Real Options; Monte Carlo Simulation; Least Squares Regression; Information Technology (IT). 


\section{Sumário}

1 Introdução

2 Investimentos em Tecnologia da Informação

2.1. Características dos Investimentos em Tecnologia da Informação 19

2.2. Avaliação de Investimentos em Tecnologia da Informação

3 Avaliação de Opções Americanas

26

3.1. Métodos Numéricos Baseados em Simulação

26

3.1.1. Método de Grant, Vora e Weeks

28

3.1.1.1. Resumo do Algoritmo

30

3.1.1.2. Determinação dos Parâmetros do Método

32

3.1.1.3. Análise de Convergência

33

3.1.2. Método dos Mínimos Quadrados de Monte Carlo 35

3.1.2.1. Exemplo Numérico - Cálculo de uma Put Americana 36

3.1.2.2. Determinação dos Parâmetros do Método 42

3.1.2.2.1. Funções Base

42

3.1.2.2.2. Número de Simulações

3.1.2.2.3. Número de Datas de Exercício

3.1.2.3. Análise de Convergência

3.2. Extensões

3.2.1. Técnicas de Redução de Variância

3.2.2. Seqüências Quase-aleatórias

3.2.3. Técnicas de Aceleração de Convergência para o Método de GVW

4 Modelos Relacionados a Investimentos em Tecnologia da Informação

4.1. Modelo de Schwartz e Zozaya (2000)

4.1.1. Modelo para Projetos de Desenvolvimento

4.1.1.1. Valor da Oportunidade de Investimento 
4.1.2. Modelo para Projetos de Aquisição 59

4.1.2.1. Valor do Ativo de TI 60

4.1.2.2. Valor da Oportunidade de Investimento 61

4.2. Modelo de Schwartz (2002) 62

4.2.1. Modelo para Projetos de Pesquisa e Desenvolvimento 63

4.2.1.1. Valor do Projeto 65

4.2.1.2. Valor da Oportunidade de Investimento 66

5 Aplicações $\quad 67$

5.1. Desenvolvimento de uma Nova Tecnologia 67

5.1.1. Solução Numérica $\quad 71$

5.1.1.1. Valor do Projeto com Opção de Abandono 72

5.1.1.2. Valor do Projeto sem Opção de Abandono 74

5.1.2. Resultados Numéricos 75

5.1.3. Análise de Sensibilidade 79

5.1.3.1. Parâmetros do Custo 79

5.1.3.2. Parâmetros do Fluxo de Caixa 82

5.1.3.3. Outros Parâmetros 85

5.2. Aquisição de um Ativo de TI 88

5.2.1. Solução Numérica $\quad 91$

5.2.1.1. Valor do Projeto com Opção de Espera 91

5.2.1.2. Valor do Projeto sem Opção de Espera 93

5.2.2. Resultados Numéricos 93

5.2.3. Análise de Sensibilidade 96

5.2.3.1. Parâmetros do Custo 96

5.2.3.2. Parâmetros do Fluxo de Caixa 99

$\begin{array}{ll}\text { 5.2.3.3. Outros Parâmetros } & 101\end{array}$

6 Conclusão 104

7 Referências Bibliográficas 106 
Apêndice A: Resultados da Análise de Sensibilidade do Projeto de $P \& D$

A.1. Resultados Numéricos 108

A.2. Gráficos Complementares 111

Apêndice B: Resultados da Análise de Sensibilidade do Projeto de Aquisição 114

B.1. Resultados Numéricos 114

B.2. Gráficos Complementares 117

Apêndice C: Réplica de Resultados de Araújo (2004) 120

Apêndice D: Réplica dos Resultados de Schwartz (2002) 122

D.1. Resultados da Simulação 123

D.2. Resultados da Análise de Sensibilidade 124

Apêndice E: Desenvolvimento Matemático 126

E.1. Modelo de Schwartz e Zozaya (2000) 126

E.1.1. Equação (4.7) da Oportunidade de Investimento do

Projeto de Desenvolvimento 126

E.1.2. Equação (4.19) da Oportunidade de Investimento do Projeto de Aquisição 129

E.2. Modelo de Schwartz (2002) 130

E.2.1. Equação (4.23) do Valor do Projeto 130

E.2.2. Equação (4.27) da Oportunidade de Investimento 132

Apêndice F: Código Fonte dos Algoritmos 135

F.1. Método Binomial para Opção Americana 135

F.2 Método de GVW para Opção Americana sobre ativo com MGB 136

F.3. Método LSM 138

F.4. Valor do Projeto de P\&D com Opção de Abandono via LSM 141

F.5. VPL do Projeto de P\&D 145

F.6. Valor do Projeto de Aquisição com Opção de Espera via LSM 147

$\begin{array}{ll}\text { F.7. VPL do Projeto de Aquisição } & 150\end{array}$ 


\section{Lista de Figuras}

Figura 1: Diagrama do RUP

Fonte: site http://www.msc-inc.net/images/rup_fu1.jpg

Figura 2: llustração dos passos do algoritmo de avaliação do

método de GVW

Figura 3: Gráfico da análise de convergência de exemplo em Araújo (2004)

Figura 4: Ranking das técnicas de redução de variância em função da velocidade de e da redução do erro de convergência

Figura 5: Classificação da homogeneidade das seqüências SMC e SQMC em função do número de dimensões do problema

Figura 6: Diagrama do projeto de TI de aquisição

Fonte: Schwartz e Zozaya (2000), p.4

Figura 7: Diagrama do projeto de TI de desenvolvimento

Fonte: Schwartz e Zozaya (2000), p.4

Figura 8: Diagrama do projeto genérico de TI

Fonte: Schwartz e Zozaya (2000), p.4

Figura 9: Simulação das variáveis de estado do exemplo do projeto de

P\&D 76

Figura 10: Histograma da percentagem de caminhos abandonados $\quad 78$

Figura 11: Percentual acumulado de abandono

Figura 12: Gráfico do custo esperado versus o valor do projeto de P\&D 80

Figura 13: Percentual de caminhos abandonados em função do custo esperado no projeto de P\&D

Figura 14: Gráfico da taxa máxima de investimento versus o valor do projeto de P\&D

Figura 15: Percentual de caminhos abandonados em relação à taxa máxima de investimento no projeto de P\&D 82

Figura 16: Gráfico do fluxo de caixa versus o valor do projeto de P\&D 83 
Figura 17: Percentual de caminhos abandonados em relação ao retorno instantâneo do fluxo de caixa no projeto de $\mathrm{P} \& \mathrm{D}$

Figura 18: Gráfico da volatilidade do fluxo de caixa versus o valor do projeto de P\&D

Figura 19: Gráfico do vencimento da patente versus o valor do

projeto de P\&D

Figura 20: Gráfico do múltiplo do fluxo de caixa versus o valor do projeto de $\mathrm{P} \& \mathrm{D}$

Figura 21: Gráfico da taxa de desconto versus o valor do projeto de P\&D

Figura 22: Gráfico da correlação entre custo e fluxo de caixa versus o valor do projeto de P\&D

Figura 23: Percentual de caminhos abandonados em relação à taxa de ocorrência de catástrofe no projeto de P\&D

Figura 24: Simulação das variáveis de estado do exemplo do projeto de aquisição de $\mathrm{TI}$

Figura 25: Diagrama da regra ótima de investimento do projeto de aquisição com opção de espera

Figura 26: Gráfico do custo esperado versus o valor do projeto de aquisição

Figura 27: Gráfico da relação entre a regra de investimento e a taxa de mudança nos custos no projeto de aquisição

Figura 28: Gráfico do custo esperado versus o valor do projeto de aquisição

Figura 29: Gráfico do fluxo de caixa por período versus o valor do projeto de aquisição

Figura 30: Gráfico da relação entre a regra de investimento e o fluxo de caixa anual no projeto de aquisição

Figura 31: Gráfico da relação entre a volatilidade do FC, o percentual de inv. adiados e o tempo médio de espera no projeto de aquisição Figura 32: Gráfico da vida útil do ativo de TI versus o valor do projeto de aquisição 
Figura 33: Gráfico da taxa de desconto versus o valor do projeto de aquisição

Figura 34: Gráfico da correlação versus o valor do

projeto de aquisição

Figura 35: Gráfico da incerteza técnica dos custos versus o valor do projeto de P\&D

Figura 36: Percentual de caminhos abandonados em relação ao valor do fluxo de caixa no projeto de P\&D

Figura 37: Gráfico do parâmetro de tendência do fluxo de caixa versus o valor do projeto de P\&D

Figura 38: Gráfico do prêmio de risco do fluxo de caixa versus o valor do projeto de P\&D

Figura 39: Gráfico da taxa de ocorrência de catástrofe versus o valor do projeto de P\&D

Figura 40: Gráfico da taxa de mudança no custo de ativos de TI versus o valor do projeto de aquisição

Figura 41: Gráfico do prêmio de risco dos custos versus o valor do projeto de aquisição

Figura 42: Gráfico do parâmetro de tendência do fluxo de caixa versus o valor do projeto de aquisição

Figura 43: Gráfico da volatilidade do fluxo de caixa versus o valor do projeto de aquisição

Figura 44: Gráfico do prêmio de risco do fluxo de caixa versus o valor do projeto de aquisição

Figura 45: Gráfico da regra de investimento em relação à taxa de desconto no projeto de aquisição

Figura 46: Influência da probabilidade de poisson no processo regido por $\mathrm{F}(\mathrm{V}, \mathrm{K})$

Figura 47: Influência da probabilidade de poisson no processo regido por $\mathrm{F}(\mathrm{C}, \mathrm{K}, \mathrm{t})$ 


\section{Lista de Tabelas}

Tabela 1: Balanço patrimonial financeiro

Tabela 2: Resultados da simulação de uma put americana através do método de GVW

Tabela 3: Matriz de simulação do preço da ação

Tabela 4: Matriz de fluxos de caixa atualizada no instante final

Tabela 5: Matriz de parada ótima atualizada no instante final

Tabela 6: Dados para a regressão no instante 2

Tabela 7: Comparação dos valores de exercício imediato e de continuação no instante 2

Tabela 8: Matriz de parada ótima atualizada no instante 2

Tabela 9: Dados para a regressão no instante 1

Tabela 10: Comparação dos valores de exercício imediato e de continuação no instante 1

Tabela 11: Matriz completa da regra ótima de exercício

Tabela 12: Matriz de fluxos de caixa realizados

Tabela 13: Análise de convergência de exemplo em Araújo (2004), em função do número de datas de exercício

Tabela 14: Relação dos parâmetros da opção simulada

Tabela 15: Resultados da simulação de uma put americana através do método LSM

Tabela 16: Parâmetros do exemplo numérico do projeto de P\&D

Tabela 17: Resultados da simulação do exemplo do projeto de P\&D

Tabela 18: Parâmetros do exemplo numérico do projeto de aquisição de um ativo de TI

Tabela 19: Resultados da simulação do exemplo do projeto de aquisição

Tabela 20: Resultados da análise de sensibilidade sobre os 
Tabela 21: Resultados da análise de sensibilidade sobre os parâmetros do fluxo de caixa

Tabela 22: Resultados da análise de sensibilidade sobre os parâmetros restantes

Tabela 23: Resultados da análise de sensibilidade sobre os parâmetros de custo

Tabela 24: Resultados da análise de sensibilidade sobre os parâmetros do fluxo de caixa

Tabela 25: Resultados da análise de sensibilidade sobre os parâmetros restantes

Tabela 26: Parâmetros da opção de venda analisada por Araújo (2004)

Tabela 27: Réplica dos resultados da simulação de Araújo (2004)

Tabela 28: Parâmetros do projeto de P\&D analisado por

Schwartz (2002)

Tabela 29: Réplica dos resultados da simulação de Schwartz (2002) 123

Tabela 30: Análise dos parâmetros de custo

Tabela 31: Análise dos parâmetros do fluxo de caixa 